\title{
Prognostic Value of Metabolic Parameters Measured By 18F-FDG PET/CT In Patients With Metastatic Cutaneous Malignant Melanoma
}

\author{
Ruihe Lai \\ Nanjing Drum Tower Hospital: Nanjing University Medical School Affiliated Nanjing Drum Tower Hospital \\ Chong Jiang \\ Nanjing Drum Tower Hospital: Nanjing University Medical School Affiliated Nanjing Drum Tower Hospital \\ Zhaoqun Chu \\ Nanjing Drum Tower Hospital: Nanjing University Medical School Affiliated Nanjing Drum Tower Hospital \\ Zhengyang Zhou ( $\square$ zyzhou@nju.edu.cn) \\ Nanjing University Medical School Affiliated Nanjing Drum Tower Hospital \\ Yue Teng \\ Nanjing Drum Tower Hospital: Nanjing University Medical School Affiliated Nanjing Drum Tower Hospital \\ Shoulin Xu \\ Nanjing Drum Tower Hospital: Nanjing University Medical School Affiliated Nanjing Drum Tower Hospital \\ Lianjun Zhao \\ Nanjing Drum Tower Hospital: Nanjing University Medical School Affiliated Nanjing Drum Tower Hospital \\ Zhengyun Zou \\ Nanjing Drum Tower Hospital: Nanjing University Medical School Affiliated Nanjing Drum Tower Hospital \\ Chongyang Ding \\ Jiangsu Province Hospital and Nanjing Medical University First Affiliated Hospital
}

\section{Original research}

Keywords: metabolic parameters, metastatic cutaneous malignant melanoma, $\mathrm{PET}$, prognosis

Posted Date: January 26th, 2021

DOI: https://doi.org/10.21203/rs.3.rs-152697/v1

License: @ (1) This work is licensed under a Creative Commons Attribution 4.0 International License. Read Full License 


\section{Abstract}

Purpose: The purpose of this study was to investigate the prognostic relevance of metabolic parameters measured using ${ }^{18} \mathrm{~F}-\mathrm{FDG}$ PET/CT in patients with metastatic cutaneous malignant melanoma (CMM).

Materials and Methods: The prognostic impact of whole-body metabolic tumor volume (wMTV), whole-body tumor lesion glycolysis (wTLG), maximum standardized uptake value (SUVmax) and mean standardized uptake value (SUVmean) was evaluated in 42 metastatic CMM patients who underwent ${ }^{18} \mathrm{~F}$-FDG PET/CT. The metabolic parameters were dichotomized by optimal cutoff values using time-dependent receiver operating characteristic (ROC) curves. In addition, univariate and multivariate analyses of the metabolic parameters were performed using the Kaplan-Meier method and Cox proportional hazards models.

Results: The optimal cutoff values for disease-free survival (DFS) were 4.63 for SUVmax, 3.31 for SUVmean, $8.22 \mathrm{~cm}^{3}$ for wMTV, and 18.22 for wTLG. The optimal cutoff values for melanoma-specific survival (MSS) were 4.77 for SUVmax, 3.31 for SUVmean, $22.32 \mathrm{~cm}^{3}$ for wMTV, and 51.37 for wTLG. Thirty-two (72\%) of the 42 patients experienced recurrence during the follow-up period, and 21 patients (50\%) died from the disease. In univariate analysis, SUVmax greater than $4.63(p=0.025)$ and SUVmean greater than $3.31(p=0.011)$ affected DFS, while SUVmax greater than $4.77(p=0.039)$, wMTV greater than $22.32(p=0.023)$ and wMTV greater than $51.37(p=0.016)$ affected MSS. In multivariate analysis after adjustment for the effects of clinical parameters, SUVmax was the best predictive factor for DFS $(p=0.016)$, and SUVmax, wMTV and WTLG were the best predictive factors for MSS $(p=0.023, p=0.018$, and $p=0.007)$.

Conclusions: SUVmax appears to be a strong independent prognostic factor for recurrence in metastatic CMM, and SUVmax, WMTV and wTLG were found to be the best predictive markers for melanoma-specific death.

\section{Introduction}

Cutaneous malignant melanoma (CMM) arises from melanocytes, which originate from the neural crest, and is responsible for $80 \%$ of skin cancer-related deaths. The incidence rate of CMM significantly increased by more than $300 \%$ between 1975 and 2012, especially in Caucasian populations[1]. It has been reported that the 5-year survival rate of malignant melanoma patients declines from $40 \%-78-5 \%-19 \%$ from stage III to stage IV $[2,3]$. Due to the poor prognosis, the accurate prognostic assessment of individual patients is important for the identification of patients at low risk of recurrence and for the selection of patients at high risk to receive intensive therapies.

FDG PET/CT is a functional imaging modality most commonly used to assess tumor glucose metabolism and proliferation. Metastatic malignant melanoma is monitored by FDG PET/CT primarily for staging and for assessing tumor recurrence and therapy response[4-6]. The standardized uptake value (SUV) is a popular index for assessing the degree of FDG accumulation in diverse cancers. Most studies with FDG PET/CT have used single pixel values of the maximum SUV (SUVmax) as an index of tumor metabolism[7, 8]. However, SUVmax represents only the most active part of the tumor and probably does not reflect the whole metabolic activity. Larson SM and Biehl KJ et al. proposed that metabolic tumor volume (wMTV) and total lesion glycolysis (WTLG) were better indexes to overcome the limitations of SUVmax and to reflect the volume of the tumor at the same time[9, 10]. To our knowledge, the prognostic significance of the FDG metabolic parameters of metastatic melanoma lesions has not been completely confirmed in CMM patients. In the present study, we investigated the prognostic significance of these parameters in the metastatic lesions of patients with CMM.

\section{Materials And Methods}

\section{Patients}

This retrospective study was approved by the institutional review board, and the requirement for written informed consent was waived. From 2012 to 2018, patients with pathologically confirmed metastatic CMM who underwent FDG PET/CT imaging were enrolled. A total of 62 patients were screened, and 20 patients were excluded (10 patients without measurable hypermetabolic lesions on the FDG PET/CT scan; 3 patients with only brain metastasis; 7 patients with a follow-up time of less than 3 months after the FDG PET/CT scan). The remaining 42 patients were included in our study. For the enrolled patients, clinical parameters such as age, sex, clinical stage, serum LDH level, treatment, location of the metastatic lesion and metabolic parameters such as SUVmax, SUVmean, wMTV, and WTLG were reviewed and retrieved. The patients underwent complete chemotherapy, immunotherapy, biotherapy or targeted therapy alone or in combination.

\section{FDG PET/CT Image Acquisition}

All patients fasted for more than $6 \mathrm{~h}$ before examination. Fasting blood glucose $\leq 10.0 \mathrm{mmol} / \mathrm{L}$ was checked before the intravenous administration of ${ }^{18} \mathrm{~F}-\mathrm{FDG}$ at a dose of $0.14 \mathrm{mCi} / \mathrm{kg}(5.18 \mathrm{MBq} / \mathrm{kg})$. Whole-body PET/CT images were performed using a Philips GEMINI GXL $\mathrm{PET} / \mathrm{CT}$ after the patients rested for $1 \mathrm{~h}$ (CT scanning parameters: tube voltage $120 \mathrm{kV}, 120 \mathrm{~mA}$, tube current $5.0 \mathrm{~mm}$, thick layer $5.0 \mathrm{~mm}$ ). After 
a low-dose CT scan without contrast from the skull to the toes with the double upper limb position on both sides of the thigh or parallel to the chest, a PET scan was obtained within the same range. The CT data were applied for attenuation correction and iterative reorganization. Images of transverse, sagittal, and coronal CT, PET and PET/CT fusion were obtained.

\section{Image Analysis}

The PET/CT images were reviewed independently by two experienced nuclear medicine physicians on a MEDEX workstation, and a final consensus was achieved for all patients. The CT scan, along with other clinical information from the patients' files, was used to help differentiate between benign and treatment-related findings and metastatic disease. The SUVmax and SUVmean were sketched automatically by the software. To assess tumor burden, MTV was determined as the volume of voxels with a threshold SUV of $40 \%$ or greater of the SUVmax[11]. wMTV was calculated as the sum of the individual MTVs of all metastatic lesions. TLG was calculated as the MTV multiplied by the SUVmean of each focal lesion in the MTV. wTLG was calculated as the sum of the TLG values of all metastatic lesions[12].

\section{Clinical End Points and Follow-up}

To evaluate recurrence, physical examination was performed every 3 months for 1 to 2 years and every 6 months to 1 year for more than two years. The patient's follow-up period was defined as the time from ${ }^{18} \mathrm{~F}$-FDG PET/CT imaging to the last clinical review. Melanoma-specific survival (MSS) was defined as the time from ${ }^{18} \mathrm{~F}$-FDG PET/CT imaging to death. Disease-free survival (DFS) was defined as the time from ${ }^{18} \mathrm{~F}-$ FDG PET/CT imaging to disease progression or death due to metastatic melanoma.

\section{Statistical Analysis}

Statistical analysis was performed by using SPSS 20.0. The optimal cutoff values of SUVmax, SUVmean, wMTV and wTLG were calculated based on the time-dependent receiver operating characteristic (ROC) curves of the subjects for the prediction of recurrence or death. Fisher's exact test was performed to analyze the survival differences between the two groups of data segmented by different metabolic parameters. The log-rank test was used to evaluate differences between Kaplan-Meier curves for DFS and MSS. Univariate analysis was used to evaluate the PET parameters and clinical variables associated with DFS and MSS. Multivariable Cox proportional hazards analysis was used to evaluate independent prognostic factors. A $p$ value of 0.05 or less was considered significant.

\section{Results}

\section{Patient Characteristics}

The clinical characteristics of the patients in this study are outlined in Table 1. A total of 42 eligible patients consisting of 27 men and 15 women with a median age of 60 years (range, 42-84 years) were enrolled in this study. Of these 42 patients, 24 (57\%) had TNM stage III CMM, and 18 (43\%) had TNM stage IV CMM. Primary lesions were located on the extremity in 33 patients (79\%) and on the trunk in 9 patients (21\%). An elevated serum LDH level ( $\geq 245 \mathrm{U} / \mathrm{L}$ ) was observed in 7 patients (17\%). Based on the location of the metastatic lesion, 32 patients (76\%) suffered from lymph node and/or skin and/or soft tissue metastasis, and 10 patients (24\%) suffered from other organ metastasis. The median survival period was 31.3 months, and the median follow-up period was 26.3 months. By the time of the analysis, 32 patients (76\%) had experienced disease progression, and 21 patients (50\%) had died from the disease. 
Demographics and Clinical Characteristics of the Study Population

\begin{tabular}{|lc|}
\hline \multicolumn{2}{|c|}{ the Study Population } \\
\hline Characteristic & \multicolumn{1}{l|}{ Value } \\
\hline Male & \\
\hline Female & $27(64 \%)$ \\
\hline Age (year) & $15(36 \%)$ \\
\hline$\leq 60$ & \\
\hline$>60$ & $21(50 \%)$ \\
\hline LDH level (U/L) & $21(50 \%)$ \\
\hline Normal<245 & $35(83 \%)$ \\
\hline Increased $\geq 245$ & $7(17 \%)$ \\
\hline Location of metastatic lesion & \\
\hline Lymph node/skin/soft tissue(L) & $32(76 \%)$ \\
\hline Other organs(O) & $10(24 \%)$ \\
\hline TNM stage & \\
\hline III & $23(21 \%)$ \\
\hline IV & $18(43 \%)$ \\
\hline Treatment & \\
\hline Single & \\
\hline Combination & \\
\hline Site of primary tumor & \\
\hline Extremity & \\
\hline Trunk & \\
\hline Abbreviations: LDH, lactate dehydrogenase \\
\hline
\end{tabular}

\section{Metabolic PET Parameters}

The medians (interquartile ranges) of SUVmax, SUVmean, wMTV, and WTLG were 6.60 (4.90-10.28), 4.07 (3.36-6.53), $8.80 \mathrm{~cm}^{3}(5.60-23.08$ $\mathrm{cm}^{3}$ ) and $30.71(13.98-88.95)$ in patients in the progression group and $4.38(3.54-8.31), 2.90(2.38-5.16), 7.87 \mathrm{~cm}^{3}\left(4.15-15.46 \mathrm{~cm}^{3}\right)$ and 14.96 (7.77-69.16) in patients in the progression-free group. SUVmax and SUVmean were higher in the progression group than in the progression-free group $(Z=-2.097, p=0.036$ and $Z=-2.028, p=0.042$, respectively). The medians (interquartile ranges) of SUVmax, SUVmean, wMTV, and wTLG were 6.17 (5.00-11.95), 4.14 (3.38-7.26), $14.42 \mathrm{~cm}^{3}\left(5.04-44.94 \mathrm{~cm}^{3}\right)$ and 59.43 (16.57-199.34) in nonsurvivors and 4.72 (3.58-7.70), $3.26(2.55-6.21), 7.28 \mathrm{~cm}^{3}\left(4.43-11.84 \mathrm{~cm}^{3}\right)$ and $16.88(10.42-39.00)$ in survivors. All five metabolic parameters in the nonsurvivor group were higher than those in the survival group $(Z=-2.704, p=0.007 ; Z=-2.579, p=0.010 ; Z=-2.000, p=0.045$ and $Z=-2.377, p=$ 0.017 , respectively).

\section{Univariate and Multivariate Survival Analyses}

The time-dependent ROC curves demonstrated that the optimal cutoff values of SUVmax, SUVmean, wMTV, and wTLG for DFS were 4.63, 3.31, $8.22 \mathrm{~cm}^{3}$, and 18.22, respectively. For MSS, the cutoff values of SUVmax, SUVmean, wMTV, and WTLG were 4.77, 3.31, 22.32 $\mathrm{cm}^{3}$, and 51.37 , respectively.

Using these cutoff thresholds, the PET parameters were dichotomized to generate Kaplan-Meier survival plots for DFS (Fig. 1) and MSS (Fig. 2). The median DFS of the good vs. poor prognostic groups dichotomized for SUVmax, SUVmean, WMTV and wTLG were 18.10 vs. 6.10 months, 13.80 vs. 4.20 months, 9.30 vs. 6.30 months, and 18.10 vs. 6.00 months, respectively. For MSS, the corresponding one- and two-year survival rates of the good vs. poor prognostic groups dichotomized for SUVmax, SUVmean, wMTV and wTLG were $86.2 \%$ vs. $58.4 \%$ and $71.1 \%$ vs. $45.5 \%, 83.3 \%$ vs. $62.0 \%$ and $63.5 \%$ vs. $48.3 \%, 77.6 \%$ vs. $50.0 \%$ and $63.1 \%$ vs. $30.0 \%$, and $81.5 \%$ vs. $50.0 \%$ and $64.9 \%$ vs. $34.3 \%$, respectively. 
In a univariate Cox proportional hazards model, SUVmax, SUVmean, location of metastatic lesion, and TNM stage were significant predictors of DFS (Table 2). The difference in DFS between patients with elevated LDH levels and those with normal levels was not significant, but a trend was noted ( $p=0.053$ ). For MSS, SUVmax, wMTV, wTLG and location of metastatic lesion were significant predictors. In a multivariate analysis including the significant clinical and PET parameters, SUVmax greater than $4.63(\mathrm{HR}, 3.032,95 \% \mathrm{Cl}, 1.234-7.451 ; \mathrm{p}=0.016)$ remained a significant independent factor associated with DFS, and SUVmax greater than $4.77(\mathrm{HR}, 3.618,95 \% \mathrm{Cl}, 1.190-11.001 ; \mathrm{p}=0.023)$, wMTV greater than 22.32 (HR, 2.892, 95\% Cl, 1.197-6.868; $\mathrm{p}=0.021)$, wTLG greater than 51.37 (HR, 3.343, 95\% Cl, 1.388-8.046; $\mathrm{p}=0.007)$ and location of metastatic lesion (other organs) (HR, 2.868, 95\% Cl, 1.180-2.883; $\mathrm{p}=0.018)$ remained significant independent factors associated with $\mathrm{MSS}$ (Table 3). 
Table 2

Univariate analysis of factors predictive of disease-free survival and melanoma-specific survival

\begin{tabular}{|c|c|c|c|c|c|c|c|c|c|c|c|c|}
\hline \multirow[t]{2}{*}{ Variable } & \multirow{2}{*}{$\begin{array}{l}\text { No. of } \\
\text { patients }\end{array}$} & \multicolumn{5}{|c|}{ Disease-free survival } & \multirow{2}{*}{$\begin{array}{l}\text { No. of } \\
\text { patient }\end{array}$} & \multicolumn{5}{|c|}{ Melanoma-specific survival } \\
\hline & & B & SE & Wald & $\begin{array}{l}\text { HR } \\
(95 \% \\
\text { Cl) }\end{array}$ & $\begin{array}{l}P \\
\text { value* }^{*}\end{array}$ & & B & SE & Wald & $\begin{array}{l}\mathrm{HR} \\
(95 \% \\
\mathrm{Cl})\end{array}$ & $\begin{array}{l}P \\
\text { value* }\end{array}$ \\
\hline Sex, M/F & $27 / 15$ & -0.381 & 0.369 & 1.067 & $\begin{array}{l}0.683 \\
(0.331- \\
1.408)\end{array}$ & 0.302 & $27 / 15$ & -0.370 & 0.485 & 0.582 & $\begin{array}{l}0.690 \\
(0.267- \\
1.788)\end{array}$ & 0.445 \\
\hline Age, $\leq 60 />60$ & $21 / 21$ & 0.231 & 0.365 & 0.402 & $\begin{array}{l}1.260 \\
(0.616- \\
2.577)\end{array}$ & 0.526 & $21 / 21$ & -0.122 & 0.154 & 0.072 & $\begin{array}{l}0.886 \\
(0.364- \\
2.155)\end{array}$ & 0.789 \\
\hline $\begin{array}{l}\text { LDH level, } \\
\text { normal/increased }\end{array}$ & $35 / 7$ & 0.863 & 0.447 & 3.736 & $\begin{array}{l}2.370 \\
(0.988- \\
5.687)\end{array}$ & 0.053 & $35 / 7$ & 0.912 & 0.492 & 3.439 & $\begin{array}{l}2.490 \\
(0.949- \\
6.530)\end{array}$ & 0.064 \\
\hline $\begin{array}{l}\text { Location of } \\
\text { metastatic } \\
\text { lesion, L*/0※ }\end{array}$ & $32 / 10$ & 0.930 & 0.392 & 5.616 & $\begin{array}{l}2.534 \\
(1.174- \\
5.466)\end{array}$ & $0.018 *$ & $32 / 10$ & 1.002 & 0.453 & 4.896 & $\begin{array}{l}2.724 \\
(1.121- \\
6.616)\end{array}$ & $0.027 *$ \\
\hline TNM stage, III/IV & $24 / 18$ & 0.802 & 0.356 & 5.073 & $\begin{array}{l}2.231 \\
(1.110- \\
4.485)\end{array}$ & $0.024^{\star}$ & $24 / 18$ & 0.591 & 0.440 & 1.803 & $\begin{array}{l}1.806 \\
(0.762- \\
4.281)\end{array}$ & 0.179 \\
\hline $\begin{array}{l}\text { Treatment, } \\
\text { single/combination }\end{array}$ & 23/19 & 0.352 & 0.362 & 0.944 & $\begin{array}{l}1.422 \\
(0.699- \\
2.893)\end{array}$ & 0.331 & 23/19 & -0.597 & 0.465 & 1.653 & $\begin{array}{l}0.550 \\
(0.221- \\
1.368)\end{array}$ & 0.199 \\
\hline $\begin{array}{l}\text { Site of primary } \\
\text { tumor, } \\
\text { extremity/trunk }\end{array}$ & $33 / 9$ & -0.152 & 0.469 & 0.105 & $\begin{array}{l}0.859 \\
(0.342- \\
2.156)\end{array}$ & 0.746 & $33 / 9$ & -0.011 & 0.524 & 0.000 & $\begin{array}{l}0.989 \\
(0.354- \\
2.765)\end{array}$ & 0.984 \\
\hline \multicolumn{13}{|l|}{ SUVmax } \\
\hline$<4.63 / \geq 4.63$ & $13 / 29$ & 1.094 & 0.433 & 6.389 & $\begin{array}{l}2.988 \\
(1.279- \\
6.981)\end{array}$ & $0.011^{\star}$ & - & - & - & - & - & - \\
\hline$<4.77 / \geq 4.77$ & - & - & - & - & - & - & $15 / 27$ & 1.152 & 0.559 & 4.249 & $\begin{array}{l}3.164 \\
(1.058- \\
9.641)\end{array}$ & $0.039 *$ \\
\hline \multicolumn{13}{|l|}{ SUVmean } \\
\hline$<3.31 / \geq 3.31$ & $18 / 24$ & 0.844 & 0.376 & 5.042 & $\begin{array}{l}2.326 \\
(1.113- \\
4.861)\end{array}$ & $0.025^{\star}$ & - & - & - & - & - & - \\
\hline$<3.31 / \geq 3.31$ & - & - & - & - & - & - & $18 / 24$ & 0.754 & 0.486 & 2.411 & $\begin{array}{l}2.126 \\
(0.821- \\
5.501)\end{array}$ & 0.121 \\
\hline \multicolumn{13}{|l|}{ wMTV $\left(\mathrm{cm}^{3}\right)$} \\
\hline$<8.22 / \geq 8.22$ & $21 / 21$ & 0.406 & 0.358 & 1.287 & $\begin{array}{l}1.501 \\
(0.744- \\
3.026)\end{array}$ & 0.257 & - & - & - & - & - & - \\
\hline$<22.32 / \geq 22.32$ & - & - & - & - & - & - & $32 / 10$ & 1.008 & 0.444 & 0.154 & $\begin{array}{l}2.739 \\
(0.148- \\
6.639)\end{array}$ & $0.023^{*}$ \\
\hline
\end{tabular}

Abbreviations: LDH, lactate dehydrogenase; SUVmax, maximum standardized uptake value; SUVmean, mean standardized uptake value; wMTV, whole-body total metabolic tumor volume; wTLG, whole-body total lesion glycolysis.

※L: Lymph node/skin/soft tissue, O: Other organs

*: $p<0.05$ 


\begin{tabular}{|c|c|c|c|c|c|c|c|c|c|c|c|c|}
\hline$<18.22 / \geq 18.22$ & $17 / 25$ & 0.697 & 0.380 & 3.367 & $\begin{array}{l}2.007 \\
(0.954- \\
4.226)\end{array}$ & 0.066 & - & - & - & - & - & - \\
\hline$<51.37 / \geq 51.37$ & - & - & - & - & - & - & $28 / 14$ & 1.052 & 0.439 & 5.757 & $\begin{array}{l}2.864 \\
(1.213- \\
6.765)\end{array}$ & $0.016^{*}$ \\
\hline \multicolumn{13}{|c|}{$\begin{array}{l}\text { Abbreviations: LDH, lactate dehydrogenase; SUVmax, maximum standardized uptake value; SUVmean, mean standardized uptake value; } \\
\text { wMTV, whole-body total metabolic tumor volume; wTLG, whole-body total lesion glycolysis. }\end{array}$} \\
\hline \multicolumn{13}{|c|}{ ※L: Lymph node/skin/soft tissue, O: Other organs } \\
\hline
\end{tabular}

Table 3

Multivariate analysis of factors predictive of survival

\begin{tabular}{|c|c|c|c|c|c|c|c|c|c|c|}
\hline \multirow[t]{2}{*}{ Variable } & \multicolumn{5}{|c|}{ Disease-free survival } & \multicolumn{5}{|c|}{ Melanoma-specific survival } \\
\hline & B & SE & Wald & $\mathrm{HR}(95 \% \mathrm{Cl})$ & $\begin{array}{l}\mathrm{P} \\
\text { value* }\end{array}$ & B & SE & Wald & $\mathrm{HR}(95 \% \mathrm{Cl})$ & $\begin{array}{l}P \\
\text { value* }\end{array}$ \\
\hline LDH level, normal/increased & -0.065 & 0.523 & 0.016 & $\begin{array}{l}0.937(0.336- \\
2.612)\end{array}$ & 0.901 & - & - & - & - & - \\
\hline $\begin{array}{l}\text { Location of metastatic } \\
\text { lesion, } L^{*} / O^{*}\end{array}$ & 0.590 & 0.460 & 1.645 & $\begin{array}{l}1.804(0.732- \\
4.443)\end{array}$ & 0.200 & 1.062 & 0.461 & 5.315 & $\begin{array}{l}2.892 \\
(1.172- \\
7.133)\end{array}$ & 0.021 * \\
\hline TNM stage, III/IV & 0.656 & 0.471 & 1.940 & $\begin{array}{l}1.927(0.766- \\
4.850)\end{array}$ & 0.164 & - & - & - & - & - \\
\hline \multicolumn{11}{|l|}{ SUVmax } \\
\hline$<4.63 / \geq 4.63$ & 1.109 & 0.459 & 5.846 & $\begin{array}{l}3.032(1.234- \\
7.451)\end{array}$ & $0.016^{*}$ & - & - & - & - & - \\
\hline$<4.77 / \geq 4.77$ & - & - & - & - & - & 1.286 & 0.567 & 5.137 & $\begin{array}{l}3.618(1.190- \\
11.001)\end{array}$ & $0.023^{*}$ \\
\hline \multicolumn{11}{|l|}{ SUVmean } \\
\hline$<3.31 / \geq 3.31$ & 0.705 & 0.404 & 3.045 & $\begin{array}{l}2.024(0.917- \\
4.466)\end{array}$ & 0.081 & - & - & - & - & - \\
\hline$<3.31 / \geq 3.31$ & - & - & - & - & - & - & - & - & - & - \\
\hline \multicolumn{11}{|l|}{ wMTV $\left(\mathrm{cm}^{3}\right)$} \\
\hline$<8.22 / \geq 8.22$ & - & - & - & - & - & - & - & - & - & - \\
\hline$<22.32 / \geq 22.32$ & - & - & - & - & - & 1.054 & 0.446 & 5.590 & $\begin{array}{l}2.868(1.197- \\
1.868)\end{array}$ & $0.018 *$ \\
\hline \multicolumn{11}{|l|}{ wTLG } \\
\hline$<18.22 / \geq 18.22$ & - & - & - & - & - & - & - & - & - & - \\
\hline$<51.37 / \geq 51.37$ & - & - & - & - & - & 1.207 & 0.448 & 7.243 & $\begin{array}{l}3.042 \\
(1.388- \\
8.046)\end{array}$ & 0.007 * \\
\hline \multicolumn{11}{|c|}{$\begin{array}{l}\text { Abbreviations: LDH, lactate dehydrogenase; SUVmax, maximum standardized uptake value; SUVmean, mean standardized uptake value; } \\
\text { SUVpeak, peak standardized uptake value; wMTV, whole-body total metabolic tumor volume; wTLG, whole-body total lesion glycolysis. }\end{array}$} \\
\hline \multicolumn{11}{|c|}{ ※L: Lymph node/skin/soft tissue, O: Other organs } \\
\hline$\star: p<0.05$ & & & & & & & & & & \\
\hline
\end{tabular}

\section{Discussion}

The application of ${ }^{18}$ F-FDG PET/CT in malignant melanoma is extensive in terms of diagnosis[13,14], stage[15,16] and therapeutic evaluation[17,18], while few prognostic studies have been reported. Tumor glycolytic activities on ${ }^{18}$ F-FDG PET have significant and independent value in predicting primary $\mathrm{CMM}[11,19]$ and metastatic malignant melanoma[12,20], while studies on metastatic CMM are rare. 
Meanwhile, regarding metabolic PET parameters, previous studies mainly focused on SUVmax, MTV (wMTV) and TLG (wTLG). In contrast, datas on the prognostic value of SUVmean in metastatic CMM are limited. In our study, all four metabolic PET parameters (SUVmax, SUVmean, WMTV and WTLG) were measured to evaluate the utility of ${ }^{18} \mathrm{~F}-\mathrm{FDG}$ PET/CT in detecting recurrence and predicting survival in patients with metastatic CMM. This retrospective study indicated that SUVmax was an independent prognostic factor for DFS and that SUVmax, WMTV and WTLG were independent prognostic factors for MSS in patients with metastatic CMM, even after adjustment for the effects of clinical parameters.

SUVmax, a well-known digitized PET parameter, has been used to evaluate the likelihood of aggressive disease, the metabolic response to therapy, the early detection of disease recurrence and prognosis. Higher SUVmax is correlated with tumor cell proliferation and poor prognosis. This study demonstrated that SUVmax was an independent prognostic factor for both DFS and MSS in patients with metastatic CMM. The only previous study including the metabolic PET parameter of SUVmax in metastatic CMM patients was that of Seban, R. D et al.[21], who reported that wMTV rather than SUVmax was the best predictive marker of MSS. They studied a cohort of 32 patients treated with immune checkpoint inhibitors (ICIs), while ours was a cohort of 42 patients treated with traditional treatment, which may cause different results. In addition, tumor shapes can be variable and asymmetrical, particularly in metastatic cancer. Furthermore, tumor composition and intratumoral FDG uptake can be heterogeneous because of cells with high or low metabolism, such as necrotic tissue or fibrotic scarring[22,23].

This study also analyzed the prognostic value of SUVmean, which is infrequently used. In a study of 80 patients with stage IIIB melanoma, Bastiaannet, E et al.[20] found that the 5-year disease-free survival rates in patients with a low mean SUV and in those with a high mean SUV were $40.9 \%$ (95\% Cl, 25.5-55.7\%) and $24.2 \%$ (95\% Cl, 12.4-38.0\%), respectively ( $p=0.02)$. The mean SUV was associated with DFS but was not associated with DSS, which was consistent with our results. These results showed that SUVmean could potentially be a useful factor in the prognostic evaluation of metastatic melanoma for both stage III and stage IV.

We concentrated on volumetric and metastatic PET parameters (wMTV, wTLG) that could evaluate patients at high risk of recurrence and death. In this study, the multivariate analysis showed that WMTV and WTLG were significant independent prognostic factors for melanoma-specific survival, even after adjusting for clinical prognostic factors in patients with metastatic CMM. wMTV and wTLG, which quantify the overall tumor burden and represent information about both tumor volume and metabolic activity, respectively[11,26], have been investigated in many tumors, such as nasopharyngeal carcinoma[27], lymphoma[28], breast cancer[29] and lung cancer[30,31]. In this study, wMTV and wTLG were the best predictive factors for MSS but not DFS. These results indicated that metastatic CMM patients with high wMTV and wTLG experience shorter overall survival, even when they have the same clinical factors. Seban, R. D et al.[21] reported 32 patients with metastatic CMM treated with immune checkpoint inhibitors targeting PD-1, and they found that the baseline WMTV and TLG were significant independent prognostic biomarkers for OS in multivariate analysis. The median OS was 28.3 (95\% Cl 8.9-44.8), and 20 out of 32 patients died. TMTV and TLG were significantly associated with poor OS (TMTV: HR 1.0, p = 0.009; TLG: HR 1.0, p = 0.009). Seban, R. D et al. also investigated whether wMTV was a significant independent prognostic biomarker for PFS, and their results were not consistent with our results. The median follow-up was 29.9 months, while ours was 38.6 months; moreover, Seban, R. D et al.'s study used treatment only targeting PD-1, while ours used single or combination treatment, which may be the reason for the different results. Ito, K. et al.[12] also emphasized the important prognostic role of wMTV in metastatic melanoma patients treated with immune checkpoint inhibitors targeting CTLA-4 (ipilimumab). They found that wMTV was a strong independent prognostic factor for OS $(p=0.001)$, which was also consistent with our results.

We recognize several limitations of this study. First, a limited number of patients were included in this retrospective study, which may explain the absence of a strong association between PET metabolic biomarkers (especially wMTV and wTLG) and PFS; therefore, further validation studies are needed in a larger cohort of metastatic CMM patients. Second, to assess tumor burden, MTV was determined as the volume enclosed by a $40 \%$ isocontour around the maximum PET voxel of the tumor lesions in this study as described by some studies, while the volume of voxels with a threshold SUV of $42 \%$ or greater of the SUVmax was also determined by other studies[11,12]. The most appropriate segmentation method for estimating MTV and TLG is still debated.

\section{Conclusion}

In conclusion, our retrospective analysis indicates that SUVmax is a strong independent prognostic factor for DFS in patients with metastatic CMM, while tumor burden, as wMTV and WTLG, is not a significant factor. WMTV, wTLG, and SUVmax are strong independent prognostic factors for MSS. Furthermore, SUVmean may be a useful imaging biomarker for evaluating the prognosis of metastatic CMM, but this findings needs further investigation.

\section{Declarations}

\section{Compliance with Ethical Standards:}

Funding: None. 
Conflict of Interest: The authors declare that they have no conflicts of interest.

Ethical approval: All procedures performed in studies involving human participants were in accordance with the ethical standards of the institutional and/or national research committee and with the 1964 Helsinki Declaration and its later amendments or comparable ethical standards.

Ethical approval: This article does not contain any studies with animals performed by any of the authors.

Informed consent: Informed consent was obtained from all individual participants included in the study.

\section{References}

1. Siegel RL, Miller KD, Jemal A.Cancer statistics, 2015. CA Cancer J Clin 2015;65(1):5-29

2. Sandru A, Voinea S, Panaitescu E, Blidaru A.Survival rates of patients with metastatic malignant melanoma.J Med Life 2014;7(4):572-576

3. Finn L, Markovic SN, Joseph RW.Therapy for metastatic melanoma: the past, present, and future. BMC Med 2012;10:23

4. Albano D, Familiari D, Fornito MC, Scalisi S, Laudicella R, Galia M, et al.Clinical and prognostic value of (18)f-fdg-pet/ct in the restaging process of recurrent cutaneous melanoma. Curr Radiopharm 2020;13(1):42-47

5. Ito K, Teng R, Schöder H, Humm JL, Ni A, Michaud L, et al.18f-fdg pet/ct for monitoring of ipilimumab therapy in patients with metastatic melanoma. J Nucl Med 2019;60(3):335-341

6. Jreige M, Letovanec I, Chaba K, Renaud S, Rusakiewicz S, Cristina V, et al. (18)f-fdg pet metabolic-to-morphological volume ratio predicts pd-11 tumour expression and response to pd-1 blockade in non-small-cell lung cancer. Eur J Nucl Med Mol Imaging 2019;46(9):1859-1868

7. Malik D, Sood A, Mittal BR, Basher RK, Bhattacharya A, Singh G.Role of (18)f-fluorodeoxyglucose positron emission tomography/computed tomography in restaging and prognosis of recurrent melanoma after curative surgery. World J Nucl Med 2019;18(2):176-182

8. Jiang C, Zhang X, Jiang M, Zou L, Su M, Kosik RO, et al.Assessment of the prognostic capacity of pretreatment, interim, and post-therapy (18)f-fdg pet/ct in extranodal natural killer/t-cell lymphoma, nasal type. Ann Nucl Med 2015;29(5):442-451

9. Larson SM, Erdi Y, Akhurst T, Mazumdar M, Macapinlac HA, Finn RD, et al.Tumor treatment response based on visual and quantitative changes in global tumor glycolysis using pet-fdg imaging. The visual response score and the change in total lesion glycolysis.Clin Positron Imaging 1999;2(3):159-171

10. Biehl KJ, Kong FM, Dehdashti F, Jin JY, Mutic S, El NI, et al.18f-fdg pet definition of gross tumor volume for radiotherapy of non-small cell lung cancer: is a single standardized uptake value threshold approach appropriate?J Nucl Med 2006;47(11):1808-1812

11. Son SH, Kang SM, Jeong SY, Lee S, Lee S, Lee J, et al.Prognostic value of volumetric parameters measured by pretreatment $18 \mathrm{f}$ fdg pet/ct in patients with cutaneous malignant melanoma.Clin Nucl Med 2016;41(6):e266-e273

12. Ito K, Schöder H, Teng R, Humm JL, Ni A, Wolchok JD, et al.Prognostic value of baseline metabolic tumor volume measured on $18 f-$ fluorodeoxyglucose positron emission tomography/computed tomography in melanoma patients treated with ipilimumab therapy.Eur $\mathrm{J}$ Nucl Med Mol Imaging 2019;46(4):930-939

13. van Wissen J, van der Hiel B, van der Hage JA, van de Wiel BA, Wouters MWJM, van Akkooi ACJ.The diagnostic value of pet/ct imaging in melanoma groin metastases.Ann Surg Oncol 2016;23(7):2323-2329

14. Zhang XX, Fang Y, Xu LB, Xu SF, Zhao ZG, Sun C, et al. [the role of preoperative (18)f-fdg pet-ct in lymphatic metastasis diagnosis of cutaneous malignant melanoma on extremities and trunk].Zhonghua Zhong Liu Za Zhi 2018;40(5):372-378

15. Twycross SH, Burger $\mathrm{H}$, Holness J.The utility of pet-ct in the staging and management of advanced and recurrent malignant melanoma.S Afr J Surg 2019;57(3):44-49

16. Dinnes J, Ferrante Di Ruffano L, Takwoingi Y, Cheung ST, Nathan P, Matin RN, et al. Ultrasound, ct, mri, or pet-ct for staging and re-staging of adults with cutaneous melanoma.Cochrane Database Syst Rev 2019;7(7):CD012806

17. Amrane K, Le Goupil D, Quere G, Delcroix O, Gouva S, Schick U, et al.Prediction of response to immune checkpoint inhibitor therapy using $18 \mathrm{f}-\mathrm{fdg}$ pet/ct in patients with melanoma.Medicine(Baltimore) 2019;98(29):e16417

18. Sachpekidis C, Kopp-Schneider A, Hakim-Meibodi L, Dimitrakopoulou-Strauss A, Hassel JC.18f-fdg pet/ct longitudinal studies in patients with advanced metastatic melanoma for response evaluation of combination treatment with vemurafenib and ipilimumab.Melanoma Res 2019;29(2):178-186

19. Kang S, Ahn BC, Hong CM, Song BI, Lee HJ, Jeong SY, et al.Can 18f-fdg pet/ct predict recurrence in patients with cutaneous malignant melanoma? Nuklearmedizin 2011;50(3):116-121

20. Bastiaannet E, Hoekstra OS, de Jong JR, Brouwers AH, Suurmeijer AJH, Hoekstra HJ.Prognostic value of the standardized uptake value for 18f-fluorodeoxyglucose in patients with stage iiib melanoma.Eur J Nucl Med Mol Imaging 2012;39(10):1592-1598

Page $9 / 11$ 
21. Seban R, Moya-Plana A, Antonios L, Yeh R, Marabelle A, Deutsch E, et al.Prognostic 18f-fdg pet biomarkers in metastatic mucosal and cutaneous melanoma treated with immune checkpoint inhibitors targeting pd-1 and ctla-4. Eur J Nucl Med Mol Imaging 2020;47(10):23012312

22. Henson JW, Ulmer S, Harris GJ.Brain tumor imaging in clinical trials. AJNR Am J Neuroradiol 2008;29(3):419-424

23. Sorensen AG, Patel S, Harmath C, Bridges S, Synnott J, Sievers A, et al.Comparison of diameter and perimeter methods for tumor volume calculation.J Clin Oncol 2001;19(2):551-557

24. Larson SM, Erdi Y, Akhurst T, Mazumdar M, Macapinlac HA, Finn RD, et al.Tumor treatment response based on visual and quantitative changes in global tumor glycolysis using pet-fdg imaging. The visual response score and the change in total lesion glycolysis.Clin Positron Imaging 1999;2(3):159-171.

25. Fei Z, Chen C, Huang Y, Qiu X, Li Y, Li L, et al.Metabolic tumor volume and conformal radiotherapy based on prognostic pet/ct for treatment of nasopharyngeal carcinoma.Medicine (Baltimore) 2019;98(28):e16327

26. Parvez A, Tau N, Hussey D, Maganti M, Metser U.(18)f-fdg pet/ct metabolic tumor parameters and radiomics features in aggressive nonhodgkin's lymphoma as predictors of treatment outcome and survival.Ann Nucl Med 2018;32(6):410-416

27. Choi JH, Lim I, Noh WC, Kim HA, Seong MK, Jang S, et al.Prediction of tumor differentiation using sequential pet/ct and mri in patients with breast cancer.Ann Nucl Med 2018;32(6):389-397

28. Pellegrino S, Fonti R, Mazziotti E, Piccin L, Mozzillo E, Damiano V, et al.Total metabolic tumor volume by $18 \mathrm{f}$-fdg pet/ct for the prediction of outcome in patients with non-small cell lung cancer.Ann Nucl Med 2019;33(12):937-944

29. Bianconi F, Palumbo I, Fravolini ML, Chiari R, Minestrini M, Brunese L, et al.Texture analysis on [(18)f]fdg pet/ct in non-small-cell lung cancer: correlations between pet features, ct features, and histological types.Mol Imaging Biol 2019;21(6):1200-1209

\section{Figures}
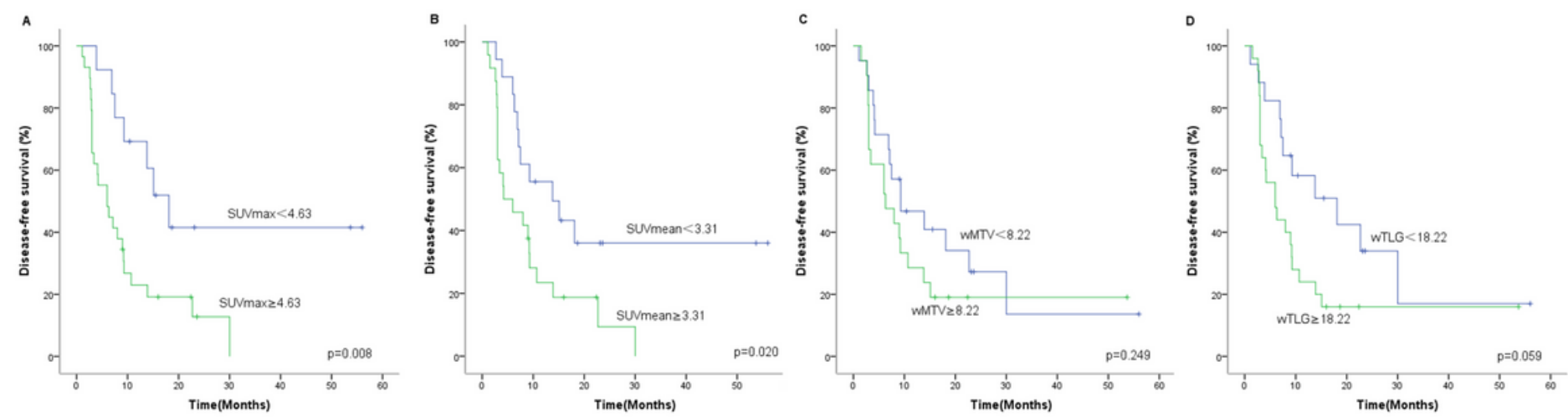

\section{Figure 1}

Kaplan-Meier analysis of the disease-free survival (DFS) of patients with metastatic cutaneous malignant melanoma (CMM) with respect to SUVmax (A), SUVmean (B), wMTV (C) and wTLG (D). SUVmax (A) and SUVmean (B) significantly affected DFS ( $p=0.008$ and $p=0.02$ ).
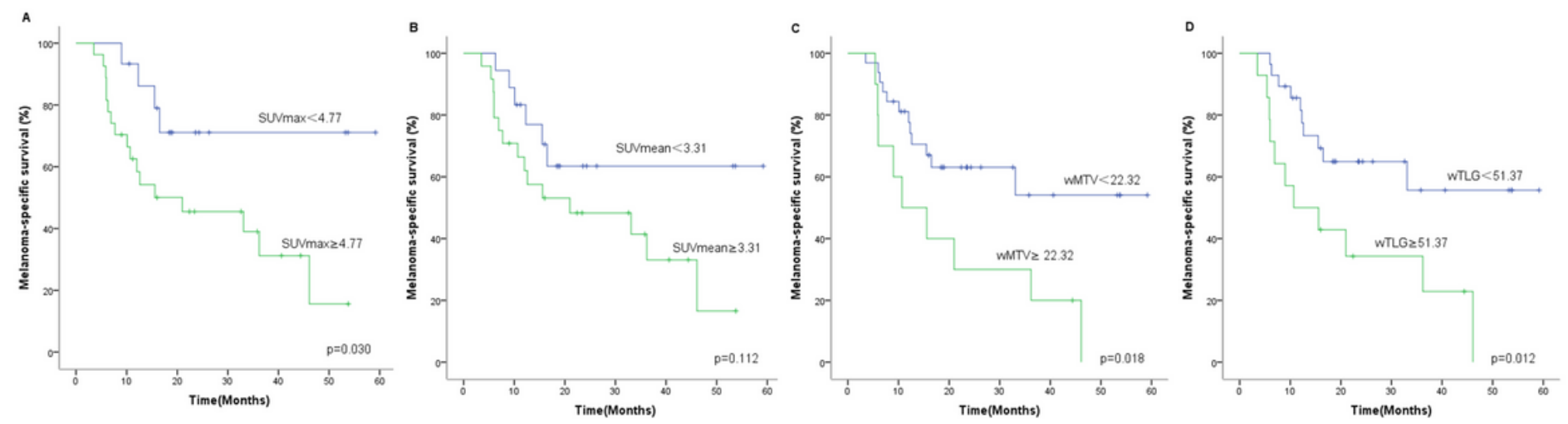

\section{Figure 2}

Kaplan-Meier analysis of the melanoma-specific survival (MSS) of patients with metastatic cutaneous malignant melanoma (CMM) with respect to SUVmax (A), SUVmean (B), wMTV (C) and wTLG (D). SUVmax (A), wMTV (C) and wTLG (D) significantly affected MSS (p=0.030, 
$p=0.018$ and $p=0.012$ ).

Page 11/11 